\title{
Effective energy densities in KrF excimer laser reformation as a sidewall smoothing technique
}

\author{
Eih-Zhe Liang \\ Department of Computer Science and Information Engineering, Diwan University, Tainan, Taiwan \\ Shih-Che Hung \\ Graduate Institute of Photonics and Optoelectronics, National Taiwan University, Taipei, Taiwan \\ Ya-Ping Hsieh \\ Department of Computer Science and Information Engineering, Diwan University, Tainan, Taiwan \\ Ching-Fuh Lin \\ Graduate Institute of Photonics and Optoelectronics, Department of Electrical Engineering, \\ and Graduate Institute of Electronics Engineering, National Taiwan University, Taipei, Taiwan
}

(Received 13 August 2007; accepted 20 November 2007; published 4 January 2008)

\begin{abstract}
Profile transformation of sidewalls and roughness reduction by $\mathrm{KrF}$ excimer laser reformation at different illumination conditions are presented. The effective energy density derived from a finite element model of heat transfer is used to characterize the molten depth of Si during high energy laser illumination at normal and oblique incidence. The operation range of laser reformation as a sidewall smoothing technique, including incident angles and energy densities, is determined by the effective energy density. Using an energy density higher than $1.4 \mathrm{~J} / \mathrm{cm}^{2}$ at the incident angle of $75^{\circ}$ is recommended for a minimal residual roughness, acceptable sidewall profile transformation, and a practical interval-height ratio. The upper bound of scattering loss after laser reformation at different effective energy densities is also calculated. (c) 2008 American Vacuum Society.
\end{abstract}

[DOI: $10.1116 / 1.2825163$ ]

\section{INTRODUCTION}

$\mathrm{KrF}$ excimer laser reformation is a novel technique for sidewall smoothing of Si waveguides. ${ }^{1}$ Among other methods, such as hydrogen annealing, ${ }^{2}$ dry oxidation, ${ }^{3}$ and wet chemical oxidation, ${ }^{4}$ it has capabilities of reducing rootmean-square (rms) roughness of Si surfaces from 14 to 0.24 $\mathrm{nm}$ and flattening protrusions as high as $100 \mathrm{~nm}$. Selective exposure for such pulsed laser treatments also allows its employment in Si photonics to integrate with electronics. ${ }^{5}$ This technique is of potential use for sidewall smoothing for integrated Si optoelectronic devices. ${ }^{6}$

The advantages of laser reformation are intriguing. Therefore, this work presents more details in this technique. Experimental results of sidewall transformation and roughness reduction at different illumination conditions are shown. A concept of effective energy density is developed to comprehensively explain the dependence of residual roughness on the incident angle. It is derived from a finite element model of heat transfer in Si during laser reformation at normal and oblique incidence. This model includes light-heat conversion at different incidence angles, heat transfer, and phase change in the Si sidewalls. The effective energy density relates the energy density at different incident angles to the molten depths. Based on the effective energy density, the operation range of energy densities at different incident angles is determined. Combined with the requirement on the intervalheight ratios of waveguides, it serves as a general guideline for selection of energy densities and incident angles for laser reformation. The upper bound of scattering loss after laser reformation at different effective energy densities is also calculated for an estimation of the influence of laser reformation on the scattering loss of $\mathrm{Si}$ waveguides.

\section{EXPERIMENT AND RESULTS}

\section{A. Laser setup and sample preparation}

The principle of laser reformation for smooth $\mathrm{Si}$ waveguides is to melt the sidewalls by a high energy laser pulse at an incident angle $(\theta)$, as illustrated in Fig. 1. The laser setup is deliberately arranged so that the incoming laser beam illuminates vertically onto the $\mathrm{Si}$ substrate, which is placed at an inclined angle. The inclined angle is equal to the incident angle of the laser beam. This configuration allows the gravitational force to act on the molten $\mathrm{Si}$ along the direction of the incident laser beam. The molten $\mathrm{Si}$ is intended to be pulled against the waveguide ridge rather than the substrate, in order to maintain the upright sidewall.

The illuminated surfaces $(\Gamma)$, including the sidewall, the top of the ridge, and the dry-etched basin, acts as heat generation sources when the absorbed light in $\mathrm{Si}$ converts to thermal energy. By laser illumination with a sufficient energy density, Si melts. ${ }^{7}$ The molten $\mathrm{Si}(\Omega)$ tends to reform, i.e., to flatten its surface by the surface tension of liquid Si to reduce the surface stress, and thus reduces the surface roughness after the reformation. The molten $\mathrm{Si}$ in a planar surface should flatten in a fashion where the surface normal is along the direction of gravitational force, as normal liquid-phase matters do. Therefore, a large inclined angle of the substrate is preferred, in order to pull the molten Si against the wave- 


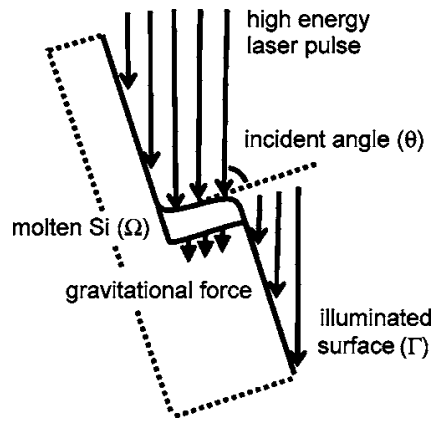

FIG. 1. Illustration of laser reformation as a sidewall smoothing technique.

guide ridge as possible. A large incident angle in such a case also provides a greater effective energy density, which is further explained by the heat transfer model in Section III.

$\mathrm{KrF}$ excimer laser (Lambda Physik) is used as the illumination source. It has a wavelength of $248 \mathrm{~nm}$, a pulse duration of $25 \mathrm{~ns}$, and an energy density up to $2 \mathrm{~J} / \mathrm{cm}^{2}$. The beam size projected on the sample is $4 \times 4 \mathrm{~mm}$. The energy density on the sample has a homogeneity of $\pm 5 \%$. The large beam size and good homogeneity are the reason to choose the excimer laser. The samples are placed in a vacuum chamber pumped to $10^{-6}$ Torr to prevent oxidation.

The waveguides are prepared as follows. An $n$-type silicon wafer with orientation (100) is spin-coated with a layer of resist (ZEP520, Zeon Chemicals) and then patterned with electron beam lithography. A chromium layer with a thickness of $50 \mathrm{~nm}$ is deposited subsequently, and the wafer is immersed in resist remover (ZDMAC, Zeon Chemicals) to lift off the residual chromium. Si ridge waveguides are fabricated by highly anisotropic reactive-ion-etch (RIE), ${ }^{8}$ using the $\mathrm{Cr}$ layer as the etching mask. Cr layer is then removed by CR-7 solution (Cyantek). The Si ridge waveguides are then placed in the vacuum chamber at an inclined angle for laser illumination.

\section{B. Sidewall transformation}

The as-etched $\mathrm{Si}$ sidewall has a rectangular shape, as shown in Fig. 2(a). The samples are tilted to three different angles, $45^{\circ}, 60^{\circ}$, and $75^{\circ}$, respectively, which are defined as incident angles on the substrate $(\theta)$, i.e., the angles between the incident laser beam and the surface normal of the $\mathrm{Si}$ substrate. They are abbreviated as incident angles in the following. The $\mathrm{KrF}$ excimer laser is used to illuminate the sidewall at $45^{\circ}, 30^{\circ}$, and $15^{\circ}$, respectively, which are defined as incident angles on the sidewall, i.e., the angles between the incident laser beam and the surface normal of the Si sidewall.

The reformed sidewalls by one shot of the laser pulse with an energy density of $1.4 \mathrm{~J} / \mathrm{cm}^{2}$ at the three incident angles are shown in Figs. 2(b)-2(d). Those illuminated by 5 shots of laser pulses with the same energy density at the three incident angles are shown in Figs. 2(e)-2(g). In general, the sidewall transforms after laser illumination in a fashion where the surface normal of the reformed sidewall is along the direction of gravitational force. At larger incident angles,
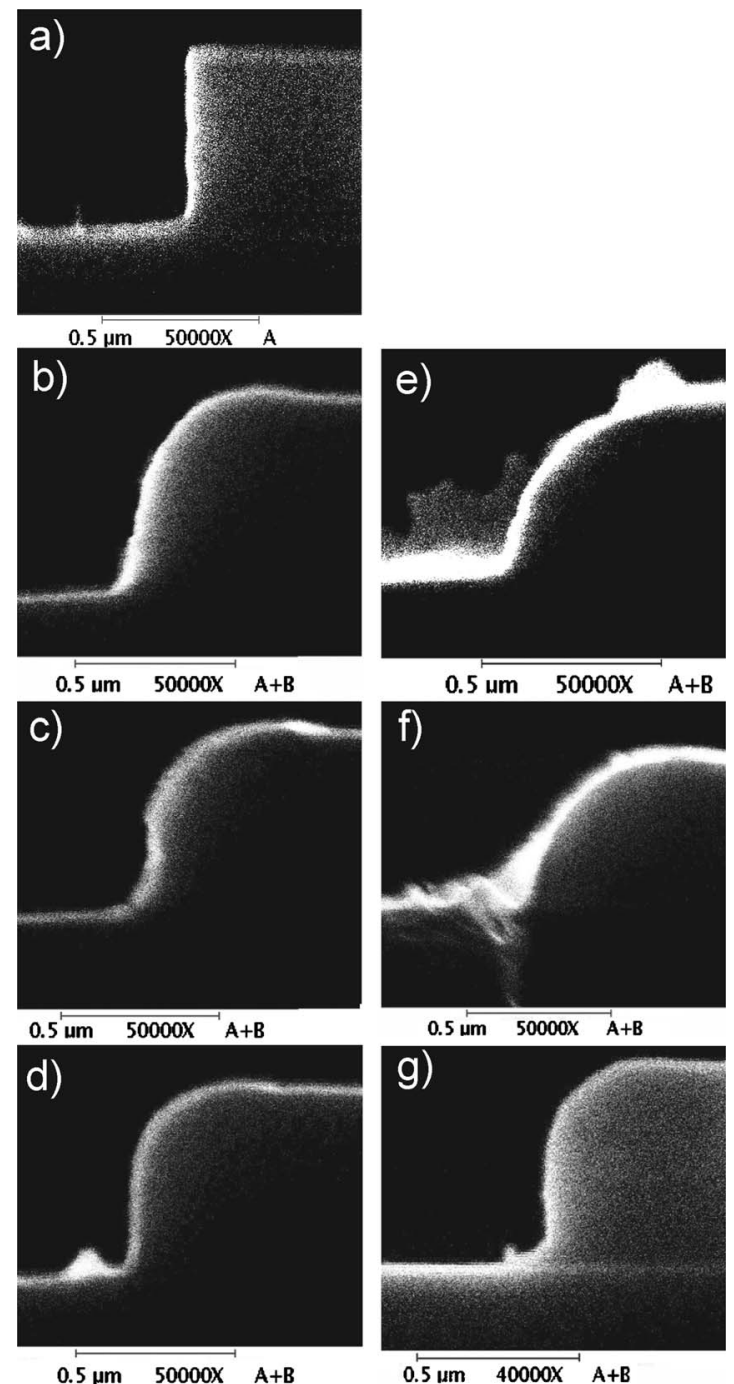

FIG. 2. SEM photos of (a) the as-fabricated in Si sidewall and the laserreformed $\mathrm{Si}$ sidewall by 1 shot of laser pulse at the incident angles of incident angles of $45^{\circ}, 60^{\circ}$, and $75^{\circ}$ in (b), (c), and (d), and by 5 shots of laser pulses at the incident angle of incident angles of $45^{\circ}, 60^{\circ}$, and $75^{\circ}$ in (e), (f), and (g), respectively.

the lower edge corners of the sidewalls are more upright from the substrate like the as-etched sidewall. At the incident angle of $75^{\circ}$, the lower corner of the sidewall is nearly vertical and it remains nearly the same as more laser pulses are illuminated.

The upper corners of the sidewalls are, however, transformed to a round corner. This region is inevitably melted since the upper corner is illuminated simultaneously at the sidewalls and at the top of the ridge. It transforms to a round shape due to the surface tension of molten $\mathrm{Si}$. They become more rounded and collapsed in the cases using 5 shots of laser pulses, especially at smaller incident angles. This is because an already transformed sidewall with a round upper corner, which has smaller incident angles on the illuminated surface, absorbs more energy density at the corner, melts deeper, and therefore continues transforming. 

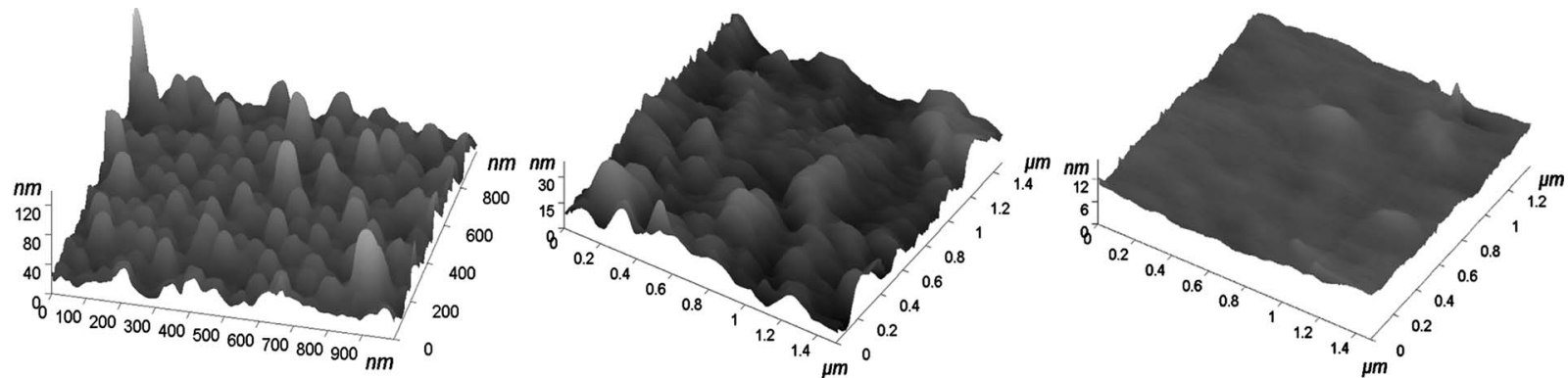

\section{a) RMS $14 \mathrm{~nm}$}

b) RMS 5.8nm
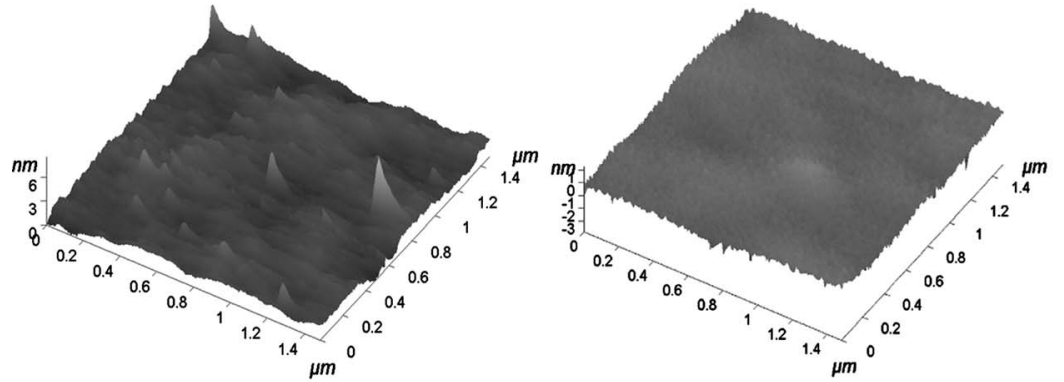

c) RMS $0.86 \mathrm{~nm}$
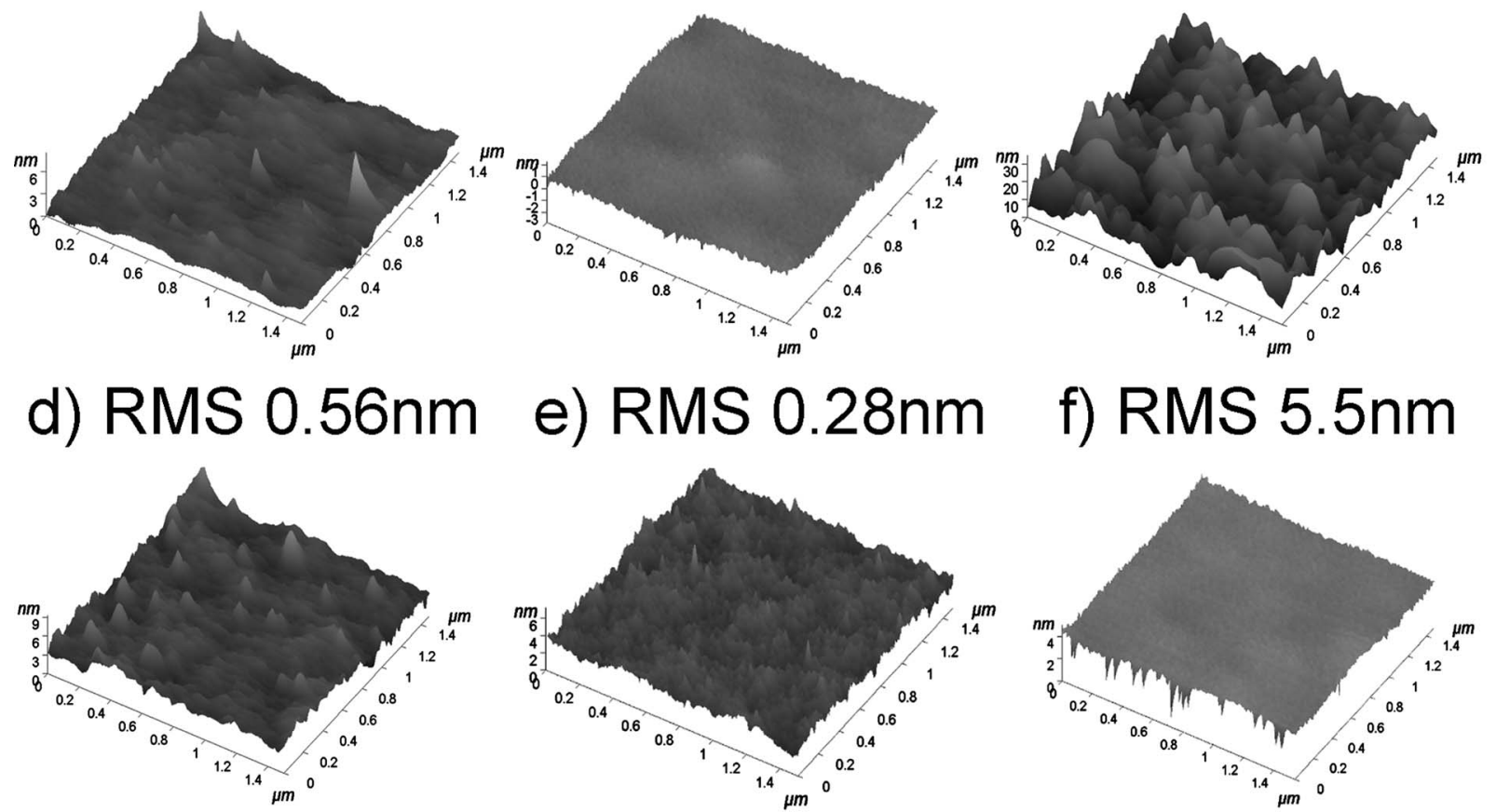

\section{g) RMS $0.81 \mathrm{~nm}$}

\section{h) RMS 0.53nm}
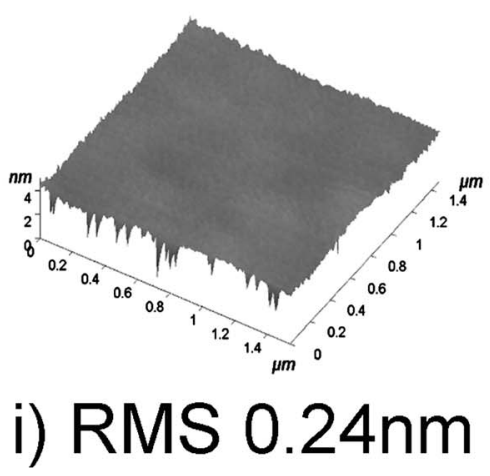

FIG. 3. AFM scans of (a) the as-etched Si surface and the laser-reformed Si surfaces at 1 shot of laser pulse at the incident angles of incident angles of $45^{\circ}$, $60^{\circ}, 75^{\circ}$, and $90^{\circ}$ in (b), (c), (d), and (e), and by 5 shots of laser pulses at the incident angle of incident angles of $45^{\circ}, 60^{\circ}, 75^{\circ}$, and $90^{\circ}$ in (f), (g), (h), and (i), respectively.

At the incident angle of $75^{\circ}$, the transformed profile by one shot of laser pulse is almost the same as that by 5 shots of laser pulses. Therefore, it is adopted in the fabrication of a smooth ridge waveguide. ${ }^{1}$

\section{Roughness reduction}

The roughness produced at the etched sidewalls comes from two origins. First is from the dry etch process. Using RIE does not produce the scallop roughness at the sidewalls like inductively coupled Plasma RIE, ${ }^{9}$ but it still produces a rough surface due to the ion bombardment. Another source of roughness comes from the $\mathrm{Cr}$ lift-off process. Evaporation of $\mathrm{Cr}$ onto the $\mathrm{Si}$ wafer generates a good deal of linewidth deviation because the thermally evaporated $\mathrm{Cr}$ aggregates to clusters. It is further transferred to the underlying $\mathrm{Si}$ and becomes a roughness along the sidewalls.
Direct surface profile measurement of the sidewalls, like atomic force microscopy (AFM), may not be exact because of the limitation of the shape of the scan tips. Therefore, laser-reformed Si planar surfaces are investigated to provide a correspondence for the laser-reformed $\mathrm{Si}$ sidewalls. A rough $\mathrm{Si}$ surface is prepared by RIE with the same etch recipe as that used to fabricate the sidewalls. The as-etched Si surface exhibits a rms roughness of $14 \mathrm{~nm}$, as shown in Fig. 3(a). Its pieces are then placed for laser illumination with incident angles of $45^{\circ}, 30^{\circ}, 15^{\circ}$, and $0^{\circ}$ on the surfaces, respectively. The energy density used is $1.4 \mathrm{~J} / \mathrm{cm}^{2}$. By 1 shot of laser pulse, the rms roughness of the as-etched surface reduces to $5.8,0.86,0.56$, and $0.28 \mathrm{~nm}$, respectively, as shown in Figs. 3(b)-3(e). By 5 shots of laser pulses, the rms roughness reduces to $5.5,0.81,0.53$, and $0.24 \mathrm{~nm}$, respectively, as shown in Figs. 3(f)-3(i). 
Some of the protrusions on the as-etched surface, as shown in Fig. 3(a), have a height exceeding $100 \mathrm{~nm}$. It is smoothed to a few nanometers after laser illumination at an energy density of $1.4 \mathrm{~J} / \mathrm{cm}^{2}$, as shown in Fig. 3(i). This result proves the capability of reducing large roughness by the laser reformation technique.

The residual roughness of the as-etched surface depends on the incident angles on the surface. At smaller incident angles on the surface, the residual roughness is smaller. The residual roughness, apparently, has to do with the difference in absorbed laser energies in the illuminated surface at different incident angles. To explain this dependence in a comprehensive manner, a finite element model of heat transfer for laser reformation is introduced in the next section.

\section{HEAT TRANSFER MODEL}

\section{A. Formulation}

In general, the same energy density of laser pulses results in different molten depths at different incident angles because of the difference in transmittance at oblique incidence. Therefore, if the sidewall is to be melted to reduce its roughness but elsewhere is not melted to prevent unexpected deformation, the energy density has to be delicately specified. Moreover, lasers used for illumination at sidewalls have various wavelength and pulse duration, which further complicates the dependence of molten depths on energy densities. In this regard, a finite element model of heat transfer is developed to determine the molten depths of Si during laser illumination at oblique incidence, given its wavelength, pulse duration, incident angles, and energy densities as the parameters.

The finite element model starts from heat generation converted from the laser pulse energy at the illuminated surfaces. To simplify the problem, the sidewall, the top of the ridge, and the dry-etched basin are described as three separate infinite planes illuminated by laser pulses at different incident angles. In such a case, the heat transfer equation for each plane is one-dimensional. Assuming absorbed light is totally converted to heat, the heat generation rate per unit area $\left(\mathrm{W} / \mathrm{cm}^{2}\right)$ at the illuminated surface is derived from the Fresnel formulas for the regime of strong absorption ${ }^{10}$ as

$$
\begin{aligned}
g(t)= & \frac{E \beta(t)}{\tau_{d}} \cos \theta_{t}\left\{r_{\perp} \frac{n_{2}}{n_{1}}\left|\frac{2 n_{1} \cos \theta_{i}}{n_{1} \cos \theta_{i}+\left(n_{2}+i \kappa_{2}\right) \cos \theta_{t}}\right|^{2}\right. \\
& \left.+r_{\|} \frac{n_{2}}{n_{1}}\left|\frac{2 n_{1} \cos \theta_{i}}{n_{1} \cos \theta_{t}+\left(n_{2}+i \kappa_{2}\right) \cos \theta_{i}}\right|^{2}\right\}
\end{aligned}
$$

where $E$ is the energy density of a single laser pulse, $\tau_{d}$ is the pulse duration, $\beta(t)$ is the normalized temporal shape of the laser pulse, $n_{1}$ is the refractive index of air, and $n_{2}+i \kappa_{2}$ is the complex refractive index of solid or liquid $\mathrm{Si}$ at the wavelength of $248 \mathrm{~nm} .^{11}$ The pulse duration is given by the integral of the normalized temporal shape of the laser pulse over time. The pulse energy is measured by an optical power meter. The pulse shape is measured by an InGaAs photodetector.

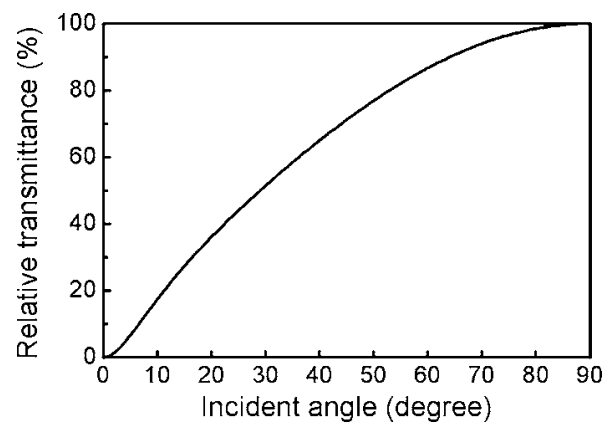

FIG. 4. Relative transmittance of $\mathrm{KrF}$ excimer laser illumination on the $\mathrm{Si}$ sidewall at different incident angles on the substrate.

The incident angle on the sidewall $\theta_{i}$ and the refractive angle $\theta_{t}$ follows Snell's law: $n_{1} \sin \theta_{i}=n_{2} \sin \theta_{t}$. The proportional ratio of light in perpendicular polarization $r_{\perp}$ and that in parallel polarization $r_{\|}$in the homogenized laser beam are assumed to be of $50 \%$ each. The relative transmittance into the $\mathrm{Si}$ sidewalls, defined as the ratio of the heat generation rate per unit area at oblique incidence to that at normal incidence, as a function of incident angles on the substrate, is shown in Fig. 4. Note that an incident angle of $\theta$ on the substrate corresponds to an incident angle of $90^{\circ}-\theta$ on the sidewall. The relative transmittance is nearly the same for solid-phase and liquid-phase Si.

The weak form of the one-dimensional heat transfer equation, which governs how the heat converted from laser pulse energy dissipates into the $\mathrm{Si}$ substrate, is derived from the differential heat transfer equation ${ }^{12}$ as

$$
\begin{aligned}
0= & -\int_{0}^{m(t)} k_{2} \frac{\partial w}{\partial x} \frac{\partial T}{\partial x} d x-\int_{m(t)}^{\infty} k_{1} \frac{\partial w}{\partial x} \frac{\partial T}{\partial x} d x \\
& +\int_{0}^{\infty}\left[w g(t)\left(2 \alpha \cos \theta_{t}\right) \exp \left(-2 \alpha x \cos \theta_{t}\right)\right. \\
& \left.-w \rho c_{v} \frac{\partial T}{\partial t}\right] d x-\frac{1}{\delta t} \int_{m(t)}^{m(t+\delta t)} w H_{l} d x,
\end{aligned}
$$

where $k_{1}$ and $k_{2}$ are the thermal conductivities of solid and liquid $\mathrm{Si}$, respectively, ${ }^{13} \rho$ is the density of $\mathrm{Si}^{13} c_{v}$ is the specific heat of $\mathrm{Si}^{13} H_{l}$ is the latent heat of liquid $\mathrm{Si}^{13}$ and $\alpha=2 \pi \kappa_{2} / \lambda$ is the absorption coefficient at the wavelength of $248 \mathrm{~nm} .{ }^{11}$ Equation (2) is a functional equation with the temperature function $T(x, t)$ as its solution and $w$ is an arbitrary weight function. The parameter $m(t)$ is the melting-front position as a function of time, which is given by

$$
T(m(t), t)=T_{\mathrm{mp}},
$$

where $T_{\mathrm{mp}}$ is the melting point of $\mathrm{Si}$. The finite element model is then formulated from Eqs. (1),(2), and (3), ${ }^{12}$ where a backward difference scheme is used and, for stability, the temperature-dependent thermal conductivity and the specific heat of Si (Ref. 13) are postulated to depend only on the temperature of past time. The melting-front position is then solved by a variant Newton's method for the finite element model. 


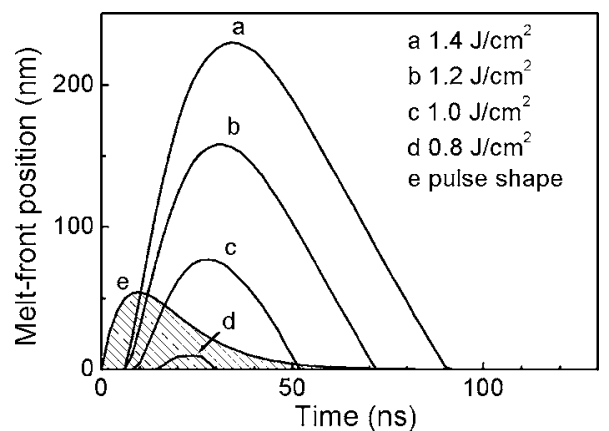

FIG. 5. Melting-front positions in Si surface illuminated with different energy densities at normal incidence.

The melting-front position of a planar Si surface illuminated with different energy densities at normal incidence is shown in Fig. 5. The shaded area is the pulse shape of the $\mathrm{KrF}$ excimer laser (with an arbitrary unit). The maximum of the melting-front position, or the molten depth, is proportional to the energy density. Using laser illumination with energy densities of $0.8,1.0,1.2$, and $1.4 \mathrm{~J} / \mathrm{cm}^{2}$, the molten depth of $\mathrm{Si}$ is calculated to be $9,78,158$, and $230 \mathrm{~nm}$, respectively. To ensure the illuminated sidewall is melted, the energy density has to exceed $0.8 \mathrm{~J} / \mathrm{cm}^{2}$ in our laser setup.

The melting-front position of the $\mathrm{Si}$ sidewall illuminated at oblique incidence with an energy density of $1.4 \mathrm{~J} / \mathrm{cm}^{2}$ at difference incident angles on the substrate is shown in Fig. 6. The molten depth corresponding to laser illumination with incident angles of $90^{\circ}, 75^{\circ}, 60^{\circ}$, and $45^{\circ}$ is $230,212,163$, and $77 \mathrm{~nm}$, respectively.

The curve (c) in Fig. 5 is nearly identical to the curve (d) in Fig. 6. It is because, from Eq. (2), the heat generation rate per unit area produced by a laser pulse with a certain energy density at oblique incidence is equivalent to that produced by a smaller energy density at normal incidence. This correspondent energy density at normal incidence is hereafter called the "effective" energy density.

Laser illumination at different incident angles with an equivalent effective energy density results in the same molten depth. As long as the absorption depth (about $10 \mathrm{~nm}$ for the wavelength of $248 \mathrm{~nm}$ ) is much smaller than the molten depth, the effective energy density can be approximated by the applied energy density at oblique incidence divided by

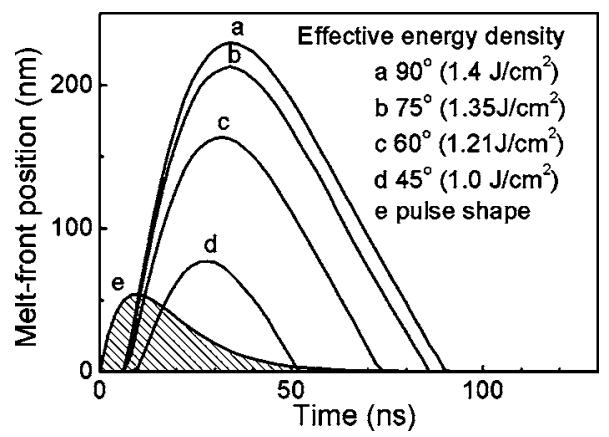

FIG. 6. Melting-front positions in Si surface illuminated at different incident angles at oblique incidence.

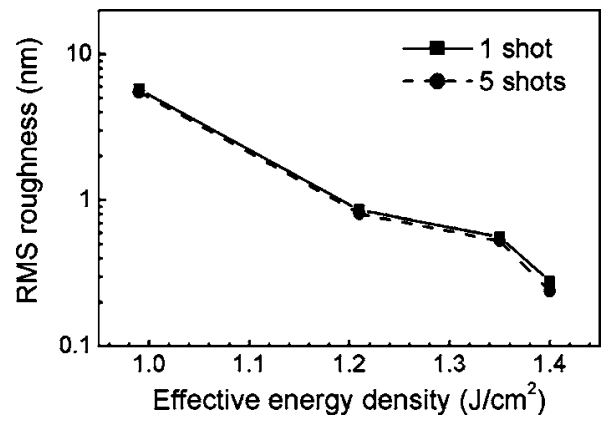

FIG. 7. Residual root-mean-square roughness of etched Si surfaces illuminated with different effective energy densities.

the relative transmittance at a given incident angle. Such a quantity proves to be useful in the roughness analysis and in the selection of energy densities to be used in laser reformation.

\section{B. Roughness analysis}

The residual roughness of reactive-ion-etched Si surface after laser reformation depends on the incident angles, as already shown in Fig. 3. The effective energy densities corresponding to the incident angles of $45^{\circ}, 30^{\circ}, 15^{\circ}$, and $0^{\circ}$ on the surface are $1.0,1.21,1.35$, and $1.4 \mathrm{~J} / \mathrm{cm}^{2}$, respectively. The relation of residual roughness to the effective energy density is depicted in Fig. 7. It is clear that using laser illumination at a large effective energy density results in a smaller rms roughness. This relation is comprehensive in the first place: a larger effective energy density results in a larger molten depth, which results in better melting of protrusions on the Si surface and then in a smoother surface.

However, the smoothing process is quite complicated. Two points are to be discussed. First, as a rule of thumb, all the protrusion on the Si surface has to be melted at least for half of its height for a smoothed surface so that the molten protrusions flow to the pores of the etched surface. Although the as-etched $\mathrm{Si}$ wafer has a rms roughness of $14 \mathrm{~nm}$, most of the protrusion has a height exceeding $100 \mathrm{~nm}$, as shown in Fig. 3(a). Considering the reduction of light transmittance into the irregular etched surface as well, an effective energy density of at least $1.0 \mathrm{~J} / \mathrm{cm}^{2}$ is required to melt all the protrusions to half of the heights. The roughness cannot be significantly reduced when the effective energy density fails to provide a sufficient molten depth.

The second point is that, even if the $\mathrm{Si}$ surface is well melted, a higher effective energy density is preferred. It provides a higher temperature above the melting point of $\mathrm{Si}$ at the molten surface, which results in a lower viscosity of molten $\mathrm{Si}^{14}{ }^{14}$ It also provides a longer time of $\mathrm{Si}$ being melted, as shown in Figs. 5 and 6. A lower viscosity allows the molten Si to flow more easily, and a longer melting duration allows the molten $\mathrm{Si}$ to flow a longer distance. As a result, even if the laser energy density is already sufficient to completely melt all the protrusions, the as-etched surface illuminated with higher effective energy densities has a smaller residual rms roughness. 
The lack of sufficient molten depth is found less influential in the smoothing process as shown in Fig. 7 because the residual roughness does not become much smaller as the number of laser pulses increases. The slight difference of residual roughness using laser illumination with 1 shot of laser pulse and 5 shots of laser pulses indicates that a lower viscosity and longer melting duration at higher effective energy density is more influential.

The heat transfer model here combines the dependence of the residual roughness on two parameters, the energy density and the incident angle, into one parameter: the effective energy density. This reduced dependence on operation parameters allows a greater selection range of illumination conditions. One may choose a smaller energy density at a larger incident angle to provide the same effective energy density for both small residual roughness and less transformed sidewall profile. The incident angle, however, is further limited by the interval-height ratio of waveguides, to be discussed in the following.

\section{Operation range}

The operation range of laser reformation, i.e., the selection of energy densities and incident angles to be used, can be evaluated for several reasons. First, residual roughness dependence on the effective energy density suggests employing a higher effective energy density. Second, higher incident angles are required for a more upright transformed profile of the waveguide. Third, in order to reduce the deformation of the corners, the sidewall is to be melted but not elsewhere. Fourth, a minimum of the interval to adjacent waveguides has to be determined to prevent shading illumination on the sidewalls by adjacent structures. The last two points will be discussed.

The top of the ridge and the dry-etched basin had better not be melted because the gravitational force intended to pull the molten sidewall also pulls these two regions. If the $\mathrm{Si}$ at the two regions is melted, unwanted deformation of the waveguide is produced. Therefore, in order not to melt the top of the ridge and the dry-etched basin, an upper bound of effective energy density is needed. The energy densities at different incident angles corresponding to this upper bound, which have effective energy densities smaller than $0.8 \mathrm{~J} / \mathrm{cm}^{2}$ at the top of the ridge and the dry-etched basin, are depicted in Fig. 8.

The lower bound for laser illumination is the requirement that sufficient energy densities have to be provided to melt the sidewall. The energy densities at different incident angles corresponding to this lower bound, which have effective energy densities larger than $0.8 \mathrm{~J} / \mathrm{cm}^{2}$ at the sidewall, are depicted in Fig. 8. Taking $\pm 5 \%$ homogenization of the laser beam into consideration, the operation range of energy densities at different incident angles is depicted as the shaded area in Fig. 8.

At large incident angles, choosing energy densities has another constraint: the interval-height ratio. It is defined to be the ratio of the interval of adjacent waveguides $(l)$ to the height of waveguides $(h)$. The relation is $l / h=\tan (\theta)$. A mini-

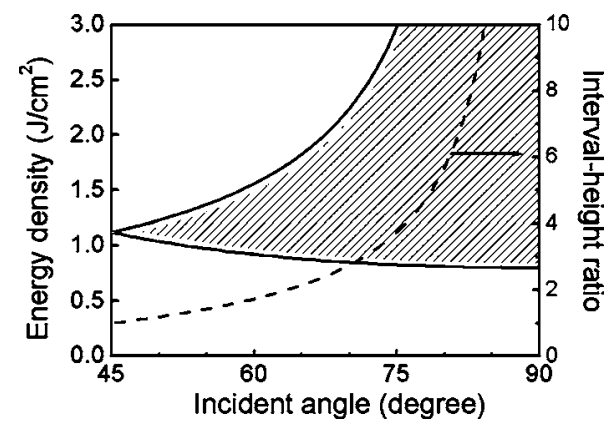

FIG. 8. Operation range of energy densities at different incident angles (shaded area) and the minimum of interval-height ratios (dashed).

mum of the interval-height ratio is required for full laser illumination of sidewalls at oblique incidence without any shading of the adjacent structures. The minimum of the interval-height ratio of repetitive waveguides is depicted as the dashed line in Fig. 8. For example, at the incident angle of $75^{\circ}$, the minimal interval-height ratio for waveguides is 3.74. In this case, a ridge waveguide with a height of $500 \mathrm{~nm}$ requires an interval of at least $1.87 \mu \mathrm{m}$ for full laser illumination. Such a minimal interval between adjacent waveguides is acceptable. At incident angles larger than $75^{\circ}$, the requirement of interval-height ratio increases nonlinearly, which is too limited for practical photonics.

Combining all four factors in the determination of the energy densities and incident angles to be used in laser reformation, an optimized selection can be made. Using an energy density higher than $1.4 \mathrm{~J} / \mathrm{cm}^{2}$ at the incident angle of $75^{\circ}$ results in a minimal residual roughness, acceptable waveguide profile transformation, and a practical intervalheight ratio of adjacent waveguides.

\section{Scattering loss}

An estimation of the scattering loss due to the sidewall roughness is the upper bound of scattering loss $\alpha_{s}$ of a single-mode waveguide with exponential statistics of roughness, which is given by ${ }^{15,16}$

$$
\alpha_{s}=0.48 \frac{\sigma^{2}}{k_{0} d^{4} n_{s i}},
$$

where $\sigma$ is the rms roughness, $k_{0}$ is the free-space wavenumber, $d$ is one-half of the width of the waveguide, and $n_{s i}$ is the refractive index of $\mathrm{Si}$ at the wavelength of the transmitted light. Suppose a fully clad waveguide fabricated by dry-etch processes and having a rms roughness of say, $14 \mathrm{~nm}$, at both sidewalls is treated by laser illumination at a certain effective energy density and the residual rms roughness at the sidewall reduces to the value as shown in Fig. 8, as the etched surfaces do. The upper bound of scattering loss due to the sidewall roughness in such a waveguide having a width of $0.5 \mu \mathrm{m}$ and transmitting light of the wavelength of $1.55 \mu \mathrm{m}$ is calculated according to Eq. (4) and shown in Fig. 9. To reduce the scattering loss due to the sidewall roughness lower than $1 \mathrm{~dB} / \mathrm{cm}$ in such a waveguide, an effective energy density of $1.2 \mathrm{~J} / \mathrm{cm}^{2}$ is required. 


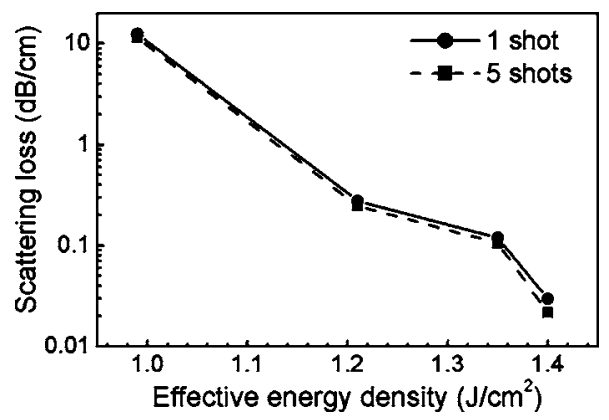

FIG. 9. Upper bounds of scattering loss with respect to laser reformation with different effective energy densities.

Although laser reformation can easily reduce the surface roughness of the sidewall, and in this way reduce the scattering loss from the sidewall, loss mechanisms such as those due to the roughness between $\mathrm{Si}$ and $\mathrm{SiO}_{2}$ interface in silicon-on-insulator waveguides and the coupling loss to the substrate and the surroundings may still present as obstacles in reduction of the total waveguide loss.

\section{CONCLUSION}

In conclusion, experimental results of $\mathrm{KrF}$ excimer laser reformation, including profile transformation of a silicon sidewall and residual roughness of reformed surfaces at different illumination conditions, are presented. The effective energy density, which is derived from the finite element model of heat transfer, is used to calculate the molten depth of Si during high energy laser illumination at normal and oblique incidence. The residual roughness of the reformed $\mathrm{Si}$ surface depends on the effective energy density. At a larger effective energy density a higher temperature in molten $\mathrm{Si}$ presents, which results in a lower viscosity and longer melting duration and thus leads to a smoother reformed surface. The operation range of laser reformation, including incident angles and energy densities, is determined based on the ef- fective energy density. An optimized condition, using an energy density higher than $1.4 \mathrm{~J} / \mathrm{cm}^{2}$ at the incident angle of $75^{\circ}$ for a minimal residual roughness, acceptable waveguide profile transformation, and a practical interval-height ratio, is recommended for smooth $\mathrm{Si}$ waveguides. An effective energy density of $1.2 \mathrm{~J} / \mathrm{cm}^{2}$ is required to reduce the scattering loss of sidewalls lower than $1 \mathrm{~dB} / \mathrm{cm}$ in a waveguide of $0.5 \mu \mathrm{m}$ width.

\section{ACKNOWLEDGMENT}

This work is supported by the National Science Council of Taiwan under Contract Number NSC 96-2218-E-434-001.

${ }^{1}$ S. C. Hung, E. Z. Liang, and C. F. Lin, in CLEO/Europe-IQEC 2007, CJ-3-TUE, Munich, Germany (2007).

${ }^{2}$ H. Kuribayashi, R. Hiruta, R. Shimizu, K. Sudoh, and H. Iwasaki, J. Vac. Sci. Technol. A 21, 1279 (2003).

${ }^{3}$ J. Takahashi, T. Tsuchizawa, T. Watanabe, and S. Itabashi, J. Vac. Sci. Technol. B 22, 2522 (2004).

${ }^{4}$ D. K. Sparacin, S. J. Spector, and L. C. Kimerling, J. Lightwave Technol. 23, 2455 (2005).

${ }^{5}$ M. C. Ferrara, M. R. Perrone, M. L. Protopapa, J. Sancho-Parramon, and S. Bosch, and S. Mazzarelli, Proc. SPIE 5250, 537 (2004).

${ }^{6}$ B. Jalali and S. Fathpour, J. Lightwave Technol. 24, 4600 (2006).

${ }^{7}$ B. C. Larson, J. Z. Tischler, and D. M. Mills, J. Mater. Res. 1, 144 (1986).

${ }^{8}$ E. Z. Liang, C. J. Huang, and C. F. Lin, J. Vac. Sci. Technol. B 24, 599 (2006).

${ }^{9}$ M.C. Lee, Y. Jin, and C.W. Ming, in 18th IEEE Conference on MEMS (2005), p. 596.

${ }^{10}$ C. F. Klingshirn, Semiconductor Optics (Springer, Berlin, 1997), Chap. 3, pp. 30-31

${ }^{11}$ L. X. Benedict, E. L. Shirley, and R. B. Bohn, Phys. Rev. B 57, R9385 (1998).

${ }^{12} \mathrm{~J}$. N. Reddy, An Introduction to the Finite Element Method, 3rd ed. (McGraw Hill, 2006), Chap. 6, pp. 292-302.

${ }^{13}$ R. F. Wood and G. E. Giles, Phys. Rev. B 23, 2923 (1981).

${ }^{14}$ H. Sasaki, E. Tokizaki, X. M. Huang, K. Terashima, and S. Kimura, Jpn. J. Appl. Phys., Part 1 34, 3432 (1995).

${ }^{15}$ F. Grillot, L. Vivien, S. Laval, D. Pascal, and E. Cassan, IEEE Photon. Technol. Lett. 16, 1661 (2004).

${ }^{16}$ F. P. Payne and J. P. R. Lacey, Opt. Quantum Electron. 26, 977 (1994). 